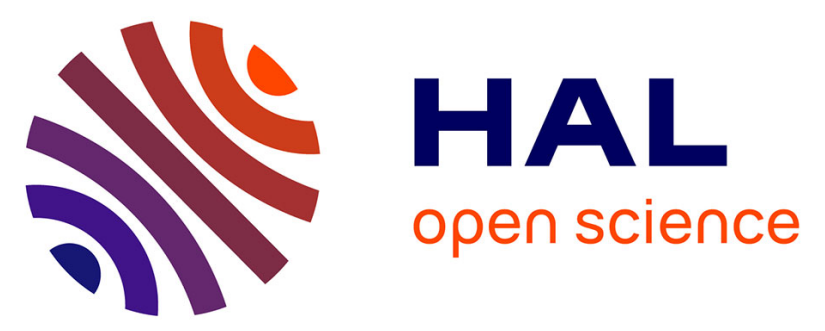

\title{
Thermal conductivity of InAs quantum dot stacks using AlAs strain compensating layers on InP substrate
} Salman Salman, Hervé Folliot, Julie Le Pouliquen, Nicolas Chevalier, Tony Rohel, Cyril Paranthoën, Nicolas Bertru, Christophe Labbé, Antoine Létoublon, Alain Le Corre

\section{To cite this version:}

Salman Salman, Hervé Folliot, Julie Le Pouliquen, Nicolas Chevalier, Tony Rohel, et al.. Thermal conductivity of InAs quantum dot stacks using AlAs strain compensating layers on InP substrate. Materials Science and Engineering: B, 2012, 177 (11), pp.882-886. 10.1016/j.mseb.2012.03.053 . hal00698574

\section{HAL Id: hal-00698574 \\ https://hal.science/hal-00698574}

Submitted on 16 May 2012

HAL is a multi-disciplinary open access archive for the deposit and dissemination of scientific research documents, whether they are published or not. The documents may come from teaching and research institutions in France or abroad, or from public or private research centers.
L'archive ouverte pluridisciplinaire $\mathbf{H A L}$, est destinée au dépôt et à la diffusion de documents scientifiques de niveau recherche, publiés ou non, émanant des établissements d'enseignement et de recherche français ou étrangers, des laboratoires publics ou privés. 


\title{
Thermal conductivity of InAs Quantum Dot stacks using AlAs strain
}

\section{compensating layers on InP substrate}

S. Salman ${ }^{1}$, H. Folliot ${ }^{1}$, J. Le Pouliquen ${ }^{1}$, N. Chevalier ${ }^{1}$, T. Rohel ${ }^{1}$, C. Paranthoën ${ }^{1}$, N.Bertru ${ }^{1}$, C. Labbé ${ }^{2}$, A. Letoublon ${ }^{1}$, A. Le Corre ${ }^{1}$

${ }^{1}$ Université Européenne de Bretagne, CNRS, Laboratoire FOTON, INSA 20 Avenue des buttes de Coësmes, 35708 Rennes, Cedex 7, France

Tel: +332232383 07, Fax: +33223238618

herve.folliot@insa-rennes.fr

${ }^{2}$ CIMAP, Centre de Recherche sur les Ions, les Matériaux et la Photonique 6 boulevard du Maréchal Juin, 14050 Caen cedex 4, France

Tel: +332314525 01, Fax +332314525 57

\begin{abstract}
The growth and thermal conductivity of InAs quantum dot (QD) stacks embedded in GaInAs matrix with AlAs compensating layers deposited on (113)B InP substrate are presented. The effect of the strain compensating AlAs layer is demonstrated through AFM and X-Ray diffraction structural analysis. The thermal conductivity $(2.7 \mathrm{~W} / \mathrm{mK}$ at $300 \mathrm{~K})$ measured by the $3 \omega$ method reveals to be clearly reduced in comparison with a bulk InGaAs layer $(5 \mathrm{~W} / \mathrm{mK})$. In addition, the thermal conductivity measurements of $\mathrm{S}$ doped $\mathrm{InP}$ substrates and the $\mathrm{SiN}$ insulating layer used in the $3 \omega$ method in the $20^{\circ} \mathrm{C}$ to $200^{\circ} \mathrm{C}$ range are also presented. An empirical law is proposed for the $\mathrm{S}$ doped InP substrate, which slightly differs from previously presented results.
\end{abstract}

\section{Keywords}

Thermoelectricity, InP substrate, InAs quantum dots, thermal conductivity

\section{Introduction}


The performance of thermoelectric materials depends on the figure-of-merit ZT, which is defined as $\mathrm{ZT}=\mathrm{S}^{2} \sigma \mathrm{T} / \lambda$, where $\mathrm{S}$ is the Seebeck coefficient, $\sigma$ the electrical conductivity, $\lambda$ the thermal conductivity, and $\mathrm{T}$ is the absolute temperature. To obtain the highest figure of merit, the power factor defined as $\mathrm{S}^{2} \sigma$ must be maximized and in the meantime the thermal conductivity must be minimized.

Several approaches have led to an enhancement of the figure of merit. Most of them have relied on the reduction of the thermal conductivity of conventional thermoelectric materials (e.g. BiTe/BiSbTe) by introducing various phonon scattering nanometre scaled defects including precipitates, grain boundaries, or superlattices. Epitaxial semiconductor nanostructures have been evidenced as very good candidates for obtaining a high figure of merit [1]. $\mathrm{Bi}_{2} \mathrm{Te}_{3} / \mathrm{Sb}_{2} \mathrm{Te}_{3}$ superlattices have been reported to have a $\mathrm{ZT} \sim 2.4$ at $300 \mathrm{~K}$ [2], while PbSeTe based quantum dots have reached a ZT up to 3 at 550K [3]. Ge QDs on Si with $3 \mathrm{~nm}$ spacing layers have also shown a clear reduction in thermal conductivity $(<1 \mathrm{~W} / \mathrm{mK})[4]$ which is a promising result for applications in thermoelectricity.

Looking at the III-V compounds family, InP and GaAs based quantum wells have fist attracted some attention for monolithic thermal cooling of laser diodes [5]. If theoretical studies on the thermoelectric properties of III-V QDs have been proposed [6,7], very little thermal conductivity experimental data of such QDs are reported to our knowledge.

Recently, high quality ErAs nano-inclusions in AlGaInAs alloys grown on InP susbtrate have been proposed and succesfully applied to thermoelectric applications. A ZT $=1.3$ at $800 \mathrm{~K}$ has been demonstrated [8]. A route not yet explored is based on stacks of Stranski-Krastanov InAs nanostructures on InP substrate grown by molecular beam epitaxy (MBE). Owing to a relative small lattice mismatch $(3.2 \%)$, different nanostructure morphologies have been evidenced in this system. On exactly oriented (100) surface, Quantum Dashes (QDashes) or quantum wires (QWires) elongated along the [1-10] direction nanostructures are easily 
obtained. The control and preservation of the morphology and the density from layer to layer when close stacking is however difficult [9].

On (113)B substrate however, well resolved QD facets are generally observed due to a higher buffer layer surface energy. This surface orientation is thus more favourable to control the stack of InAs QDs with reproducible morphologies from layer to layer. For instance, a stack of 150 InAs QD layers with 20nm spacers grown by MBE has been realised [10].

Moreover, it is well known that strain accumulation during QD stack growth leads to surface degradation, or more severely, creation of defects, such as misfit dislocations. Dislocations are known to reduce the thermal conductivity [11] when present in rather high density, but are also responsible of a reduction of carrier mobility and sometimes also act as deep levels or conductive carrier channels. These dislocations are thus generally detrimental to thermoelectric performances of semiconductor materials.

Therefore, the stack of self assembled QDs generally requires a strain compensation technique.

We here show that by balancing the compressively strained Stranski-Krastanow InAs quantum dots using AlAs tensile strained layers, a high number of closely stacked quantum dot layers with good quality can be obtained.

In the following, the $3 \omega$ thermal conductivity measurement method is briefly presented and first applied to $\mathrm{Si}$ and $\mathrm{InP}$ substrate. The insulating $\mathrm{Si}_{\mathrm{x}} \mathrm{N}_{\mathrm{y}}$ layer is also characterized in terms of thermal conductivity and interface thermal resistance. The growth of InAs QD stacks using AlAs as strain compensating layers is then presented. AFM imaging is used for structural analysis on two stacks of 20 QD layers, with and without insertion of the strain compensating layers. X-Ray analysis is used to analyse the structural quality of a stack of 100 QD layers and Photoluminescence (PL) measurement is performed to reveal the optical quality of the layers. 
The thermal conductivity of this stack is measured and shows a clear reduction when

\section{compared to a bulk lattice-matched GaInAs.}

ZT figure of merit of InAs/InP nanostructures for thermoelectric generation is finally discussed.

\section{Thermal conductivity measurements using the $3 \omega$ method}

\subsection{Experimental details}

The thermal conductivity measurements were performed using the so called " $3 \omega$ method" developed by Cahill [12] with a modified model by Borca-Tasciuc to take into account the finite thickness of the substrate and adiabatic boundary conditions [13]. This method has proven to be well suited for epitaxial films. Basically, it consists in measuring the temperature rise in a metal line driven by an $\mathrm{AC}$ current, which simultaneously acts as a heater and a thermometer, as a function of the current frequency.

All samples were prepared using the same method. Typically, a $200 \mathrm{~nm}$ silicon nitride $\left(\mathrm{Si}_{\mathrm{x}} \mathrm{N}_{\mathrm{y}}\right)$ insulating layer was deposited by PECVD (Plasma Enhanced Chemical Vapor Deposition) at $250^{\circ} \mathrm{C}$. A $\operatorname{Ti}(20 \mathrm{~nm}) / \mathrm{Au}(200 \mathrm{~nm})$ was subsequently deposited by e-beam evaporation and liftoff processed, resulting into a $10 \mathrm{~mm}$ long and $20 \mu \mathrm{m}$ wide heating metallic stripe with two $2 \times 2 \mathrm{~mm}$ pads at both ends for electrical access. The metal stripe resistance versus temperature measurements was calibrated for each metal stripe. Temperature was measured both by $\mathrm{K}$ type thermocouple and by measuring the calibrated metal line resistance.

The $3 \omega$ signal acquisition was performed using a 12 bit DAC converter in the $10 \mathrm{~Hz}$ to $1 \mathrm{kHz}$ range, using a four probe configuration. A $1 \Omega$ shunt resistance in series with the metal line was used to monitor the current. The current amplitudes were kept in the 70 to $80 \mathrm{~mA}$ for all samples, ensuring a small mean temperature elevation $(<2 \mathrm{~K})$. 


\subsection{Validation on Si silicon substrate}

In order to check the accuracy of the thermal conductivity measurements and analysis, we first used a $\mathrm{p}$ Boron $\left(\sim 10^{15} \mathrm{~cm}^{-3}\right)$ doped silicon substrate. A value of $148 \pm 1 \mathrm{~W} / \mathrm{m} . \mathrm{K}$ at $300 \mathrm{~K}$ is obtained, which is in very good agreement with previously reported values [14]. Fig. 1 shows the measured temperature rise as a function of twice the current frequency ( $2 \mathrm{f}$ ), that confirms the good agreement of our measurements with the theoretical model of BorcaTasciuc and al [0] even at rather low frequencies. The temperature elevation at low frequencies due to the finite thickness $(330 \mu \mathrm{m})$ of the Si substrate is well reproduced by the model.

Figure 1: $3 \omega$ method measured and modelled temperature elevation of a TiAu stripe on silicon substrate.

\subsection{Thermal conductivity of InP $\mathrm{n}$ doped substrate and SiN layer}

A set of four InP $\mathrm{n}=4.10^{18} \mathrm{~cm}^{-3} \mathrm{~S}$ doped (100) commercial substrates from the same ingot (supplier: Sumitomo Corporation) was used. On each of them a SiN PECVD layer with different thicknesses varying from $100 \mathrm{~nm}$ to $600 \mathrm{~nm}$ was deposited in order to characterise both $\mathrm{InP}$ and $\mathrm{SiN}$ thermal conductivity, and the total $\mathrm{TiAu} / \mathrm{SiN} / \mathrm{InP}$ interface thermal resistance.

The thermal conductivity measurements on all substrates gave similar results with small differences (within $1 \mathrm{~W} / \mathrm{mK}$ at each temperature). Fig. 2 represents the temperature dependence of the InP measured thermal conductivity. A fitting empirical power relation of the form $\lambda=\mathrm{A}(\mathrm{T} / 300)^{-\mathrm{n}}$ with $\mathrm{n}=1.57$ is deduced and found to be in good agreement with literature [15], but it is to be noticed that the A mean value at $300 \mathrm{~K}(75.6 \mathrm{~W} / \mathrm{mK})$ is slightly higher than those previously reported $(68 \mathrm{~W} / \mathrm{mK}$ [16] and $60.5 \mathrm{~W} / \mathrm{mK}$ [15]) which could be due to a higher quality of currently available substrates. 
Figure 2: $\mathrm{S}$ doped InP substrates measured thermal conductivity as a function of temperature.

The thermal conductivity of the SiN layer was also characterized and found to be $0.72 \mathrm{~W} / \mathrm{m} . \mathrm{K}$ at room temperature. As shown on Fig. 3, the thermal conductivity slightly increases at higher temperature, which is an usual characteristic of $\mathrm{SiN}$ amorphous material. These values are in very good agreement with $[17,18]$ for PECVD SiN layers. The thermal interface resistance (sum of the $\mathrm{Ti}-\mathrm{Au} / \mathrm{SiN}$ and $\mathrm{SiN} / \mathrm{InP}$ interface resistances) was also deduced by measuring the variation of thermal resistance as a function of the $\mathrm{SiN}$ layer thickness on InP samples. Measurements are presented in Fig. 4. It was found to be $4.10^{-8} \mathrm{~W} / \mathrm{m}^{2} \mathrm{~K}$ which is comparable to previously reported values for SiN layers on Si samples [18].

Figure 3: thermal conductivity ( $\lambda$ in $\mathrm{W} / \mathrm{mK}$ ) of PECVD SiN on InP as a function of temperature.

Figure 4: measured PECVD SiN thermal resistance versus SiN thickness and extracted parameters.

\section{Growth, structural and thermal conductivity characterisation of InAs stacked QDs on InP (113)B}

\subsection{Growth}

All samples were grown by Solid-Source MBE at $480^{\circ} \mathrm{C}$ on InP (113)B Sulphur (S) doped substrates (3 to $\left.5.10^{18} \mathrm{~cm}^{-3}\right)$. A single QD layer of $2 \mathrm{ML}$ of InAs $(3.2 \%$ compressively strained) on lattice matched $\operatorname{In}_{0.53} \mathrm{Ga}_{0.47}$ As was grown to check the formation of QDs. A QD area density of $5.110^{10} / \mathrm{cm}^{2}$ is obtained as shown on the AFM image (Fig. 5). QD mean height and diameter are $3 \mathrm{~nm}$ and $33 \mathrm{~nm}$ respectively. Two samples containing a stack of 20 QD layers were then grown. The last QD layer was left uncapped for AFM measurements. The first sample consisted in 20 InAs QD layers separated by $10 \mathrm{~nm}$ of lattice-matched 
InGaAs. The second differed from the first by the insertion of a $2 \mathrm{ML}$ AlAs layer in the middle of the $10 \mathrm{~nm}$ InGaAs layer. This AlAs layer, which is 3.5\% tensile strained on InP is expected to act as a strain compensating layer in order to conserve a good quality growth front [10].

Figure 5: 1x1 $\mu \mathrm{m}^{2}$ AFM image of a single layer of InAs QDs grown on InGaAs buffer on InP (113)B.

\subsection{Structural and optical characterisations}

Fig. 6. a) (without strain compensating layer) and b) (with strain compensation layer) show the AFM image of the corresponding samples. The absence of the strain compensating layer results in a very rough surface with no clear distinction of QDs, whereas its presence preserves both the shape and the density of InAs QDs with however a small undulation of the growth front. The areal density is $5.010^{10} / \mathrm{cm}^{2}$ and the average height and diameter are 40nm and $3.8 \mathrm{~nm}$ respectively. While the areal density is preserved from 1 to 20 layers, both average diameter and height are increased by $\sim 20 \%$, which likely indicates an impact of the strain field on the morphology of the dots, as already observed in [10] for thicker (20nm) spacing layers.

Figure 6: a) $1 \times 1 \mu \mathrm{m}^{2} \mathrm{AFM}$ image of the surface of a 20 stacked InAs $2 \mathrm{ML}$ layers without strain compensation layer and b) with AlAs strain compensation layer.

A sample containing a stack of 100 layers of InAs QDs with AlAs strain compensating layers with a final 200nm InP cap was subsequently grown for thermal conductivity measurements. The sample was uniformly $\mathrm{n}$ doped with $\mathrm{Si}\left(5.10^{18} \mathrm{~cm}-3\right)$ for future Seebeck measurements. The structure of the layer was investigated using $\mathrm{x}$-ray diffraction reciprocal space mapping (RSM). Measurements along the [311] (growth axis) direction (Fig. 7) show a limited but well defined number of satellite peaks showing a rather good structural quality. The extracted period is $11.35 \mathrm{~nm}$ in good agreement with the nominal $11.17 \mathrm{~nm}$ value. The mean strain status 
of the structure, obtained by the position of the diffraction peak close to the substrate peak, is found to be $0.06 \%$ in compression. At this value and for this total thickness of the structure, it is expected that very few dislocations should be created [19]. Moreover, no dislocation lines are visible on the AFM images.

Figure 7: X-Ray $\omega-2 \theta$ curve extracted from RSM along the growth axis (113) of the 100 strain compensated InAs QD stack. Thick blue vertical line is related to InP substrate, thinner vertical lines correspond to satellites peaks.

Photoluminescence (PL) was performed at 10K using a 1064nm YAG laser and the spectrum is reported in Fig. 8. At this excitation wavelength, the whole stack PL is rather uniformly excited due to the relatively small absorption coefficient of InGaAs (the absorption length is estimated to be $0.5 \mu \mathrm{m})$. The observed PL is thus representative of the whole structure. Two gaussian peaks can be resolved, and can be tentatively attributed to the InGaAs QW superlattice with AlAs barriers (peak at $0.8 \mathrm{eV}$ ) and the InAs QDs (peak at $0.7 \mathrm{eV}$ ). Simple calculation of the PL peaks using k.p. method assuming 1D confinement [20] lead to corresponding energies of $0.83 \mathrm{eV}$ and $0.66 \mathrm{eV}$ respectively, as shown in the inset of fig. 8 . PL intensity is comparable with the one of InGaAs quantum wells with AlInAs barriers lattice matched on InP, which reveals no clear degradation of the optical quality of the QD stack when AlAs strain compensating layers are inserted.

Figure 8: 10K PL spectrum of 100 InAs QD stack with strain compensation AlAs layers with two fitting Gaussian peaks. Inset: band alignment of InAs/InGaAs/AlAs system with corresponding calculated energy levels (CB: conduction band minimum at $\Gamma$ point, $\mathrm{HH}$ : heavy hole and LH: light hole band maxima). $\mathrm{E}_{1}$ and $\mathrm{HH}_{1}$ are the calculated QD ground states in the conduction and valence band respectively.

\subsection{Thermal conductivity measurements}


The stack of 100 strain compensated InAs QD layers was characterised by means of the $3 \omega$ method and compared with a $1 \mu \mathrm{m}$ thick Si doped $\left(5.10^{18} \mathrm{~cm}^{-3}\right)$ lattice matched InGaAs reference layer. The thermal conductivity of the QD stack was found to be $2.7 \pm 0.1 \mathrm{~W} / \mathrm{mK}$ at $300 \mathrm{~K}$ and to remain constant (within estimated experimental error) in the $20^{\circ} \mathrm{C}$ to $200^{\circ} \mathrm{C}$ range (Fig. 9).

Figure 9: Thermal conductivity of 100 InAs QD stack with strain compensation AlAs layers versus temperature.

The thermal conductivity of the InGaAs reference layer was measured to be $5.1 \pm 0.2 \mathrm{~W} / \mathrm{mK}$, consistent with previously reported values [21]. A higher reduction of the thermal conductivity of InGaAs / InGaAsP MQW structure has been reported for a 15/5 nm period [22]. The presented results show however an abnormal high thermal conduction (higher than bulk value) or very small period leaving unclear the origin of the observed reduction in the thermal conductivity.

If dislocations can have an impact on the reduction of thermal conductivity, their effect is expected to play a major role when their density exceeds $10^{7} / \mathrm{cm}^{2}$ as calculated in [23] and observed in for SiGe system [24] or measured in [25] for bulk GaN. To our knowledge no data is available for the InGaAs system, but we can expect that dislocations in our sample play a minor effect in the reduction of the thermal conductivity. InGaAs/AlInAs digital alloy with both comparable (1.9 $\left.10^{18} / \mathrm{cm}^{3}\right) \mathrm{n}$ doping and average composition was studied [26] and the measured thermal conductivity was only slightly reduced $(4.5 \mathrm{~W} / \mathrm{mK})$ as compared to InGaAs.

The clear reduction by a factor of $\sim 1.9$ of the thermal conductivity can thus be attributed to the presence of the QDs and AlAs strain compensating layers. The precise role of the shape, density and strain distribution inside/outside the QDs is however still unclear a would require further investigations. For instance, interdiffusion can play an important role on the thermal 
conductivity, and the precise nature of the different interfaces is required to get a clearer understanding [27].

This reduction in thermal conductivity at room temperature is comparable with the value obtained at maximum ZT of ErAs clusters in AlGaInAs on InP [8], where ErAs clusters mainly act as phonon diffusion centres, but also as electron filters.

It is interesting to note that in the same temperature range, the thermal conductivity of this material is also found to be mainly constant for 3\% ErAs concentration.

Seebeck and electrical conductivity measurements of our InAs QD stacks are in progress.

\section{Conclusions}

We have presented results on the reduction of thermal conductivity by the insertion of densely stacked InAs QDs layers embedded in lattice-matched InGaAs on InP substrate using AlAs strain compensating layers. A reduction by a factor of 1.9 of the thermal conductivity is observed at room temperature as compared to a bulk lattice-matched GaInAs layer. This reduction in thermal conductivity is similar to the best reported values on InP using ErAs nanoparticles embedded in AlGaInAs alloys on InP [02] when optimized ZT is reached.

Moreover, a revised empirical law for InP S doped substrate thermal conductivity is proposed, which could also be of interest for other applications.

Seebeck and conductivity measurements are currently under investigation.

\section{Acknowledgements}

The author wishes to thank the Région Bretagne for financial support through ARED PhD research grant.

\section{References}


[1] Paothep Pichanusakorn and Prabhakar Bandaru, Nanostructured thermoelectrics, Mat. Sci.

Eng. R: Reports 67, 2-4 (2010), 19-63.

[2] Venkatasubramanian, Rama, Edward Siivola, Thomas Colpitts, et Brooks O’Quinn. Thinfilm thermoelectric devices with high room-temperature figures of merit, Nature 413, 6856 (2001), 597-602.

[3] Harman, T. C., M. P. Walsh, B. E. laforge, et G. W. Turner, Nanostructured thermoelectric materials, J. of Elect. Mat. 34, 5 (2005), L19-L22.

[4] Pernot, G., M. Stoffel, I. Savic, F. Pezzoli, P. Chen, G. Savelli, A. Jacquot, et al, Precise control of thermal conductivity at the nanoscale through individual phonon-scattering barriers, Nat. Mat. 9, 6 (2010), 491-495.

[5]Zhang, Jizhi, Neal G Anderson, et Kei May Lau, AlGaAs superlattice microcoolers, Appl. Phys. Lett. 83, 2 (2003), 374-376.

[6] Fomin, V. M., et P. Kratzer, Thermoelectric transport in periodic one-dimensional stacks of InAs/GaAs quantum dots, Phys. Rev. B 82, 4 (2010), 045318.

[7] A. Yadav, K. P. Pipe, W. Ye, et R. S. Goldman, Thermoelectric properties of quantum

dot chains, J. of Appl. Phys. 105, (2009) 093711.

[8] J. M. O. Zide et al., High efficiency semimetal/semiconductor nanocomposite thermoelectric materials, J. App. Phys. 108, 12 (2010), 123702.

[9] Notzel Sritirawisarn, Nut, et Richard Nötzel, InAs/InP Quantum Dots, Dashes, and Ordered Arrays, Jap. J. of Appl. Phys. 48, 4 (2009), 04C121. 
[10] Ryuji Oshima et al., Optical properties of stacked InAs self-organized quantum dots on InP (3 1 1)B, J. Cryst. Growth 301-302, 0 (2007), 776-780.

[11] Watling, Jeremy R, et Douglas J Paul, A study of the impact of dislocations on the thermoelectric properties of quantum wells in the Si/SiGe materials system, J. of Appl. Phys. 110, $11(2011), 114508$.

[12] David G. Cahill, Thermal conductivity measurement from 30 to $750 \mathrm{~K}$ : the $3 \omega$ method, Rev. Sci. Instrum. 61, 2 (1990), 802.

[13] T. Borca-Tasciuc, A. R. Kumar, and G. Chen, Data reduction in $3 \omega$ method for thin-film thermal conductivity determination, Rev. Sci. Instrum. 72, 4 (2001), 2139.

[14] M. G Burzo, P. L Komarov, and P. E Raad, Non-contact thermal conductivity measurements of p-doped and n-doped gold covered natural and isotopically-pure silicon and their oxides, in Proceedings of the 5th International Conference on Thermal and Mechanical Simulation and Experiments in Microelectronics and Microsystems, 2004, EuroSimE 2004 IEEE, (2004), 269- 276.

[15] Sajan D George et al., Thermal characterization of intrinsic and extrinsic InP using photoacoustic technique, J. Phys. D Appl. Phys. 36, 8 (2003), 990-993.

[16] M. Bertolotti et al., New photothermal deflection method for thermal diffusivity measurement of semiconductor wafers, Rev. Sci. Instrum. 68, 3 (1997), 1521.

[17] S.-M. Lee and David G. Cahill, Heat transport in thin dielectric films, J. App. Phys. 81, 6 (1997), 2590. 
[18] A. J. Griffin, F. R. Brotzen, et P. J. Loos, The effective transverse thermal conductivity of amorphous Si3N4 thin films, J. App. Phys. 76, 7 (1994), 4007.

[19] Salviati, G., C. Ferrari, L. Lazzarini, L. Nasi, A.V. Drigo, M. Berti, D. De Salvador, M. Natali, et M. Mazzer, Structural characterization of InGaAs/InP heterostructures grown under compressive and tensile stress, Appl. Surf. Sc. 188, 1-2 (2002), 36-48.

[20] H. Folliot, S. Loualiche, B. Lambert, V. Drouot, et A. Le Corre, Effects of interfacelayers composition and strain distribution on the optical transitions of InAs quantum dots on InP, Phys. Rev. B 58, 16 (1998), 10700-10704.

[21] Woochul Kim et al., Cross-plane lattice and electronic thermal conductivities of ErAs:InGaAs/nGaAlAs superlattices, Appl. Phys. Lett. 88, 24 (2006), 242107.

[22] Chen Zhen, Juekuan Yang, Ping Zhuang, Minhua Chen, Jian Zhu, et Yunfei Chen, Thermal conductivity measurement of InGaAs/InGaAsP superlattice thin films, Chin. Sci. Bull. 51, 23 (2006), 2931-2936.

[23] Watling Jeremy R, et Douglas J Paul, A study of the impact of dislocations on the thermoelectric properties of quantum wells in the Si/SiGe materials system, J. App. Phys. 110, $11(2011), 114508-114508-7$.

[24] S. -M Lee, David G Cahill, et Rama Venkatasubramanian, Thermal conductivity of SiGe superlattices, Appl. Phys. Lett. 70, 22 (1997), 2957-2959.

[25] C. Mion, J. F Muth, E. A Preble, et D. Hanser, Accurate dependence of gallium nitride thermal conductivity on dislocation density, Appl. Phys. Lett. 89, 9 (2006), 092123-092123-3. 
[26] Je-Hyeong Bahk, Zhixi Bian, Mona Zebarjadi, Joshua M. O. Zide, Hong Lu, Dongyan $\mathrm{Xu}$, Joseph P. Feser, et al., Thermoelectric figure of merit of $\left(\operatorname{In}_{0.53} \mathrm{Ga}_{0.47} \mathrm{As}\right)_{0.8} /\left(\operatorname{In}_{0.52} \mathrm{Al}_{0.48} \mathrm{As}\right)_{0.2}$ III-V semiconductor alloys, Phys. Rev. B 81, $\mathrm{n}^{0} .23$ (2010), 235209.

[27] Konstantinos Termentzidis, Patrice Chantrenne, Jean-Yves Duquesne, et Abdelhak Saci, Thermal conductivity of GaAs/AlAs superlattices and the puzzle of interfaces, J. Phys. : Condens. Matter 22 (2010), 475001. 


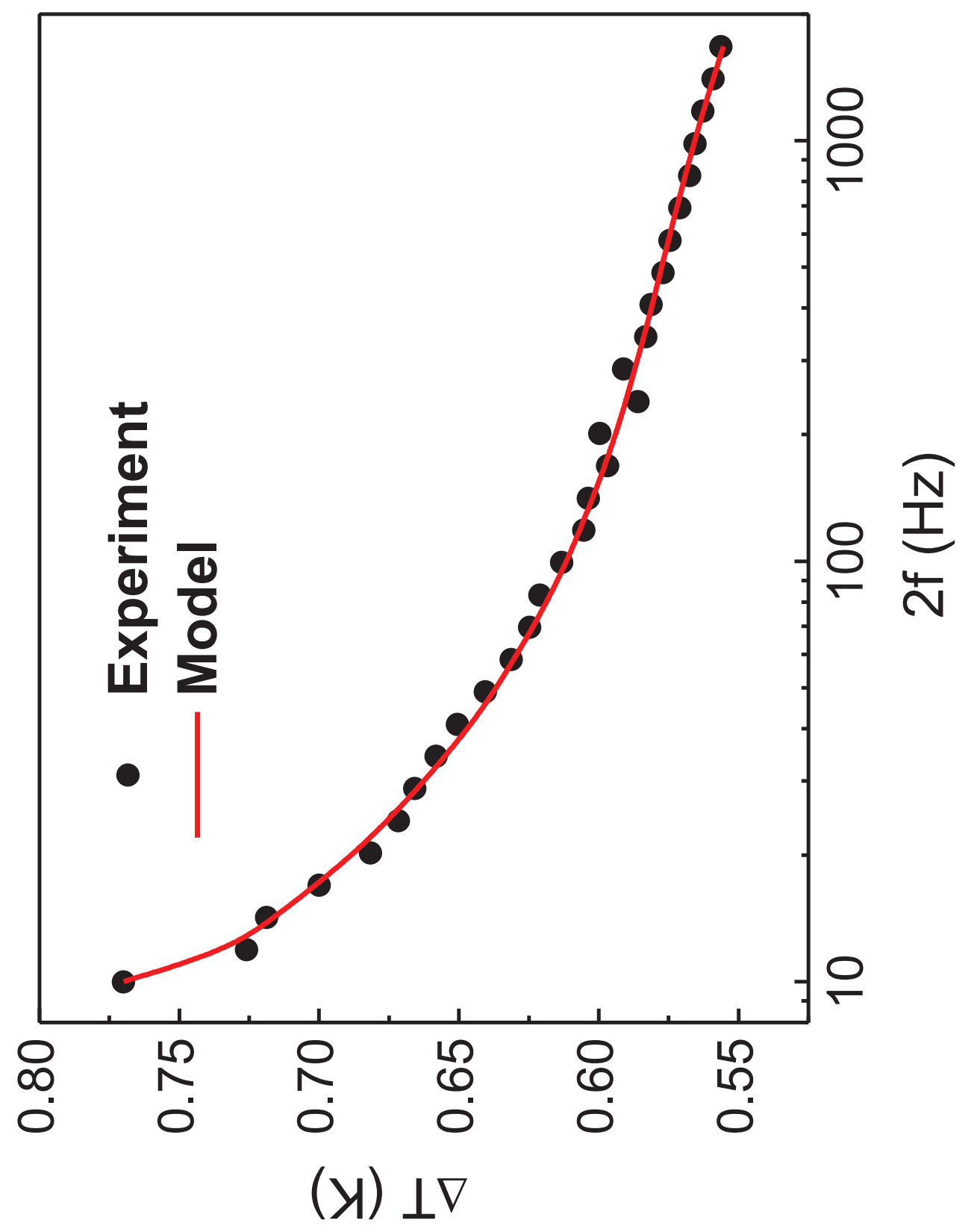




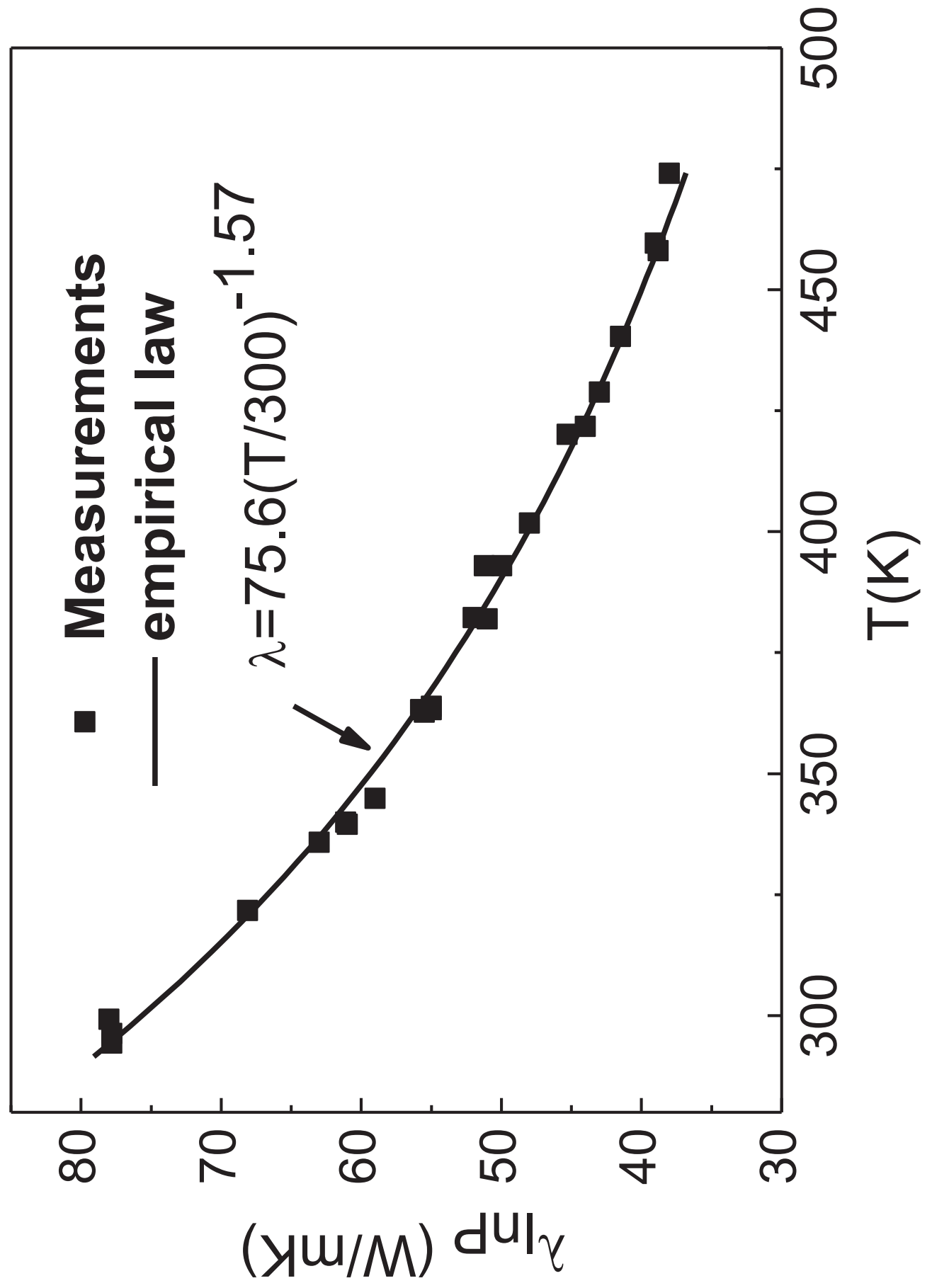

\begin{tabular}{l}
$\frac{\pi}{0}$ \\
$\frac{0}{5}$ \\
$\frac{\sigma}{i}$ \\
\hline
\end{tabular} 


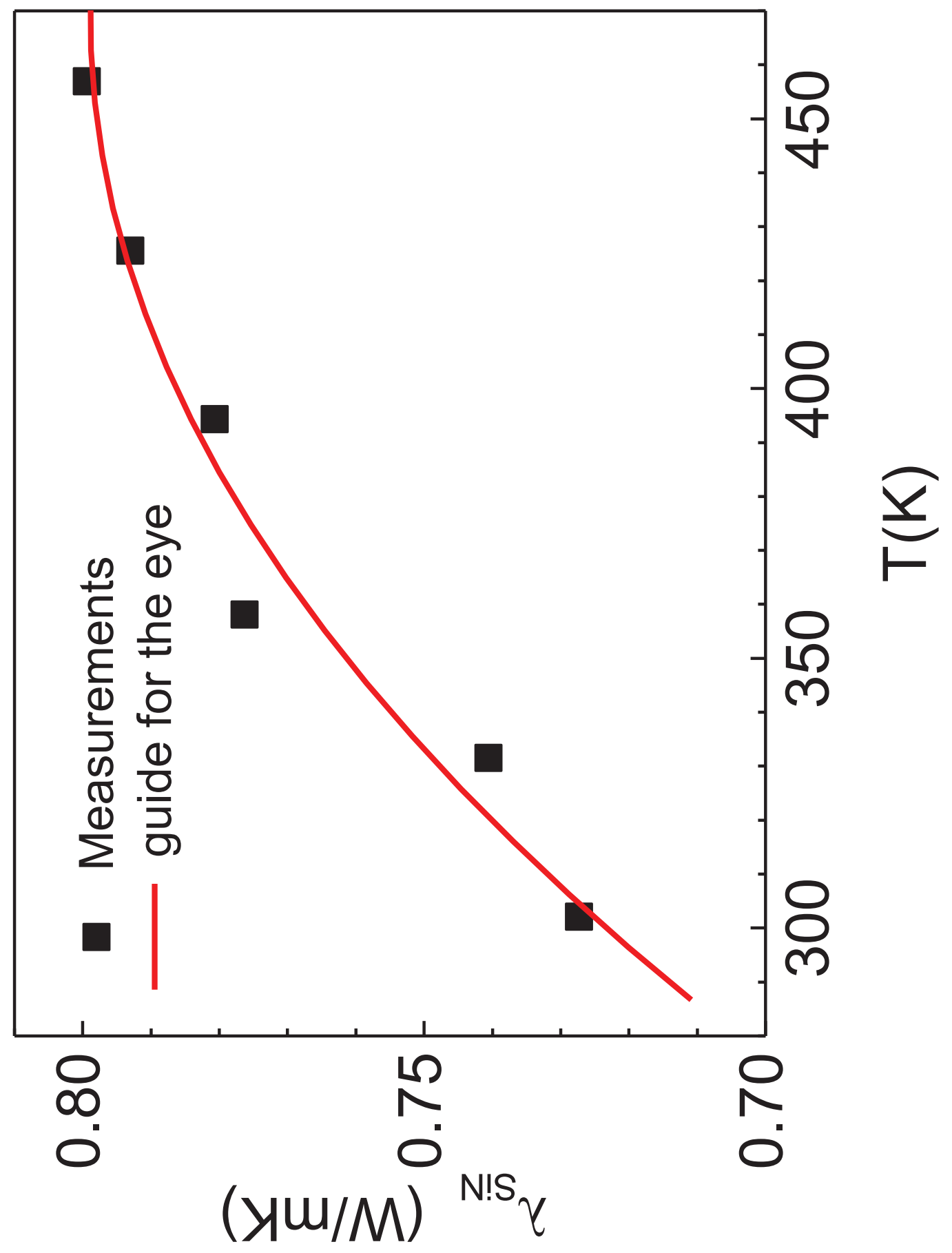




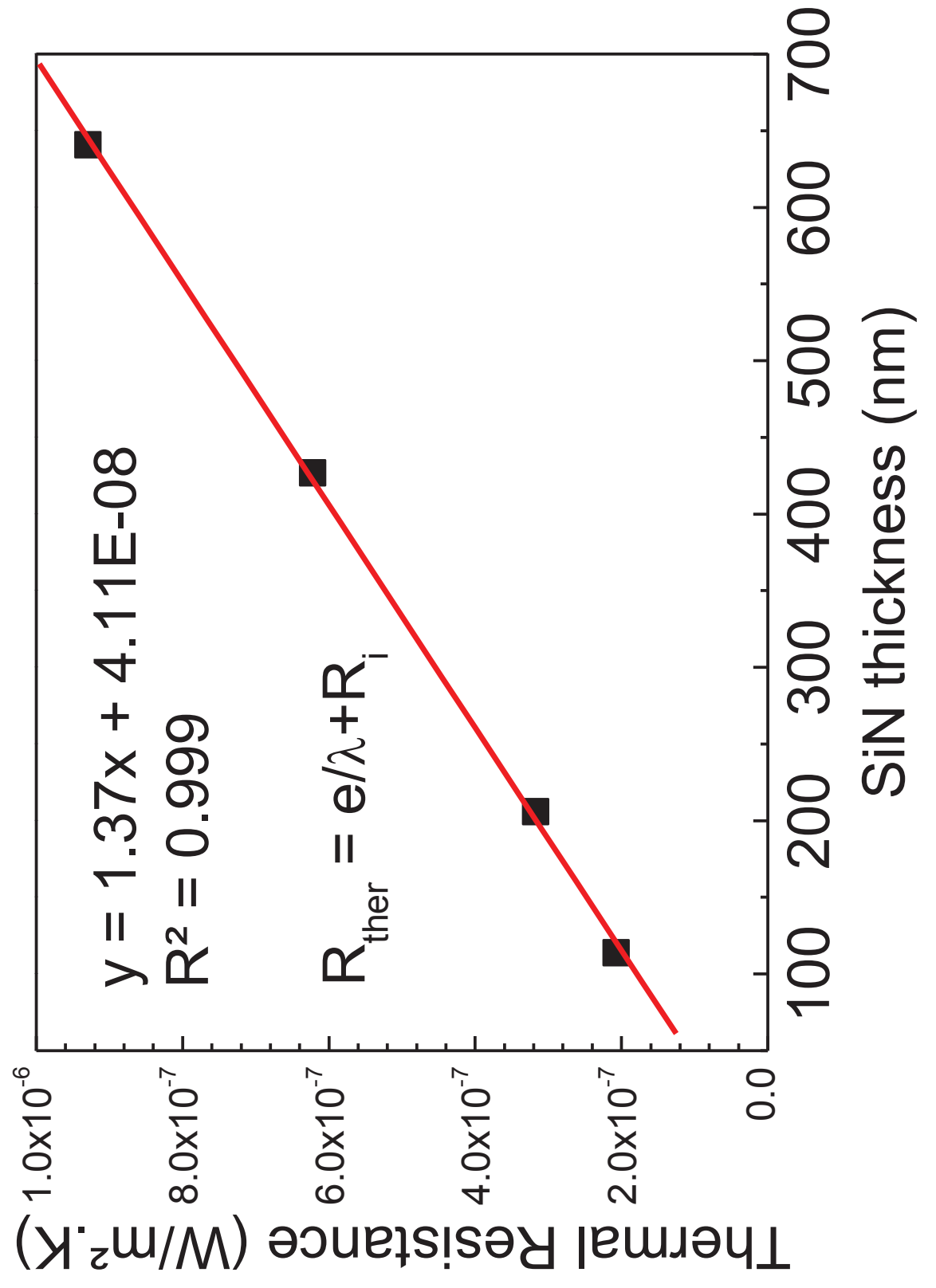

$\frac{\widehat{0}}{\frac{0}{0}}$ 


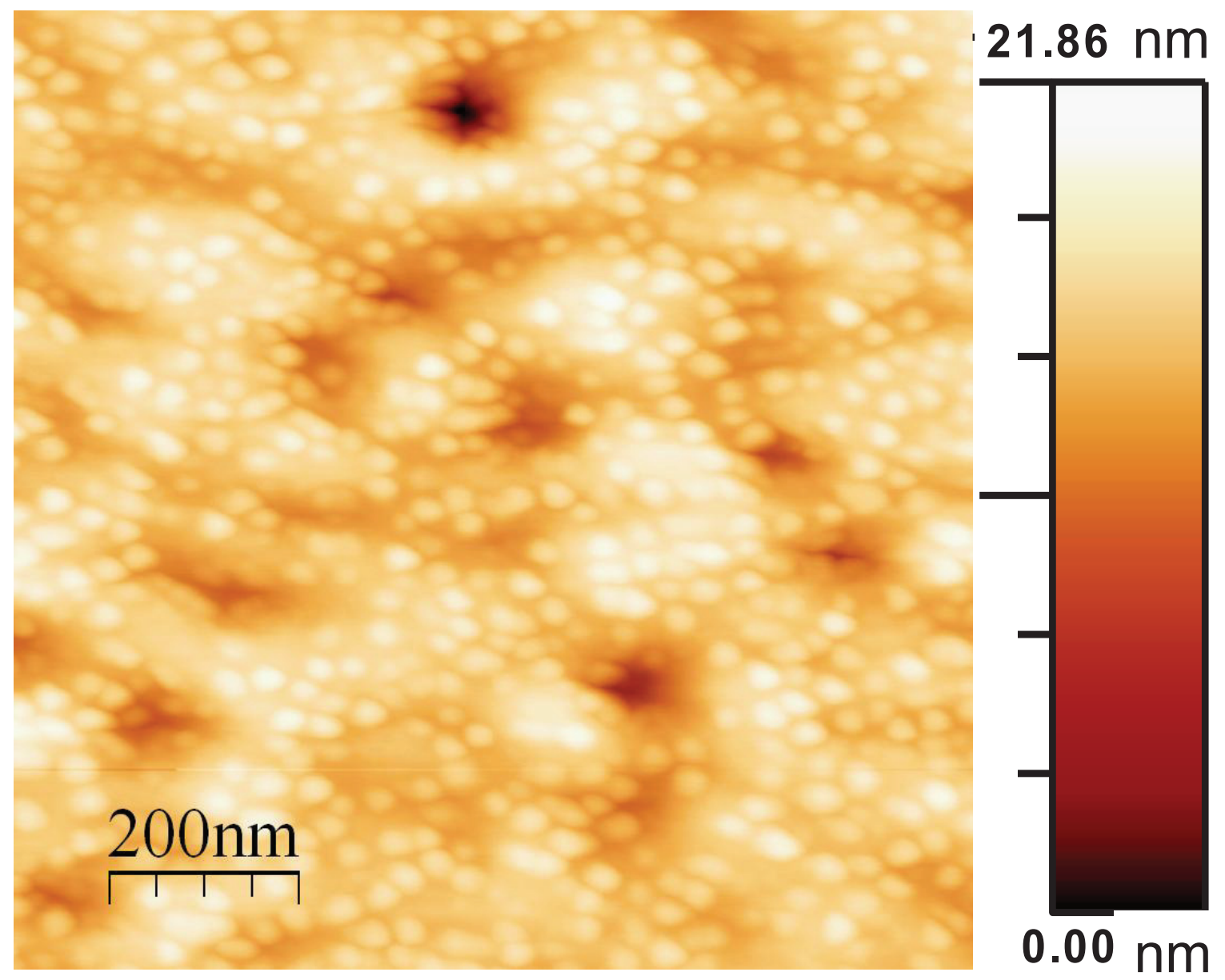



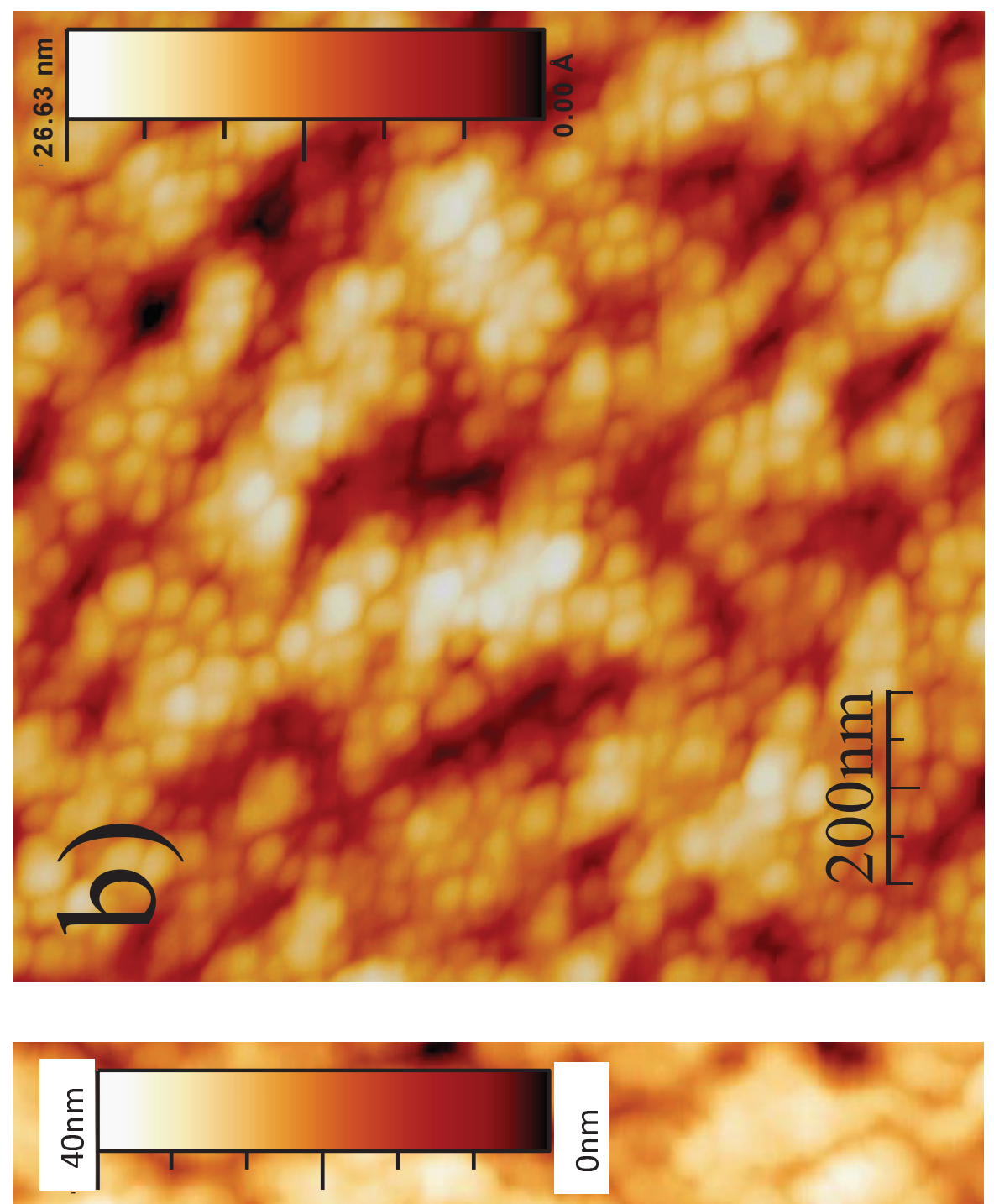

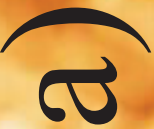

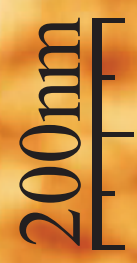

$\frac{\pi}{0}$ 


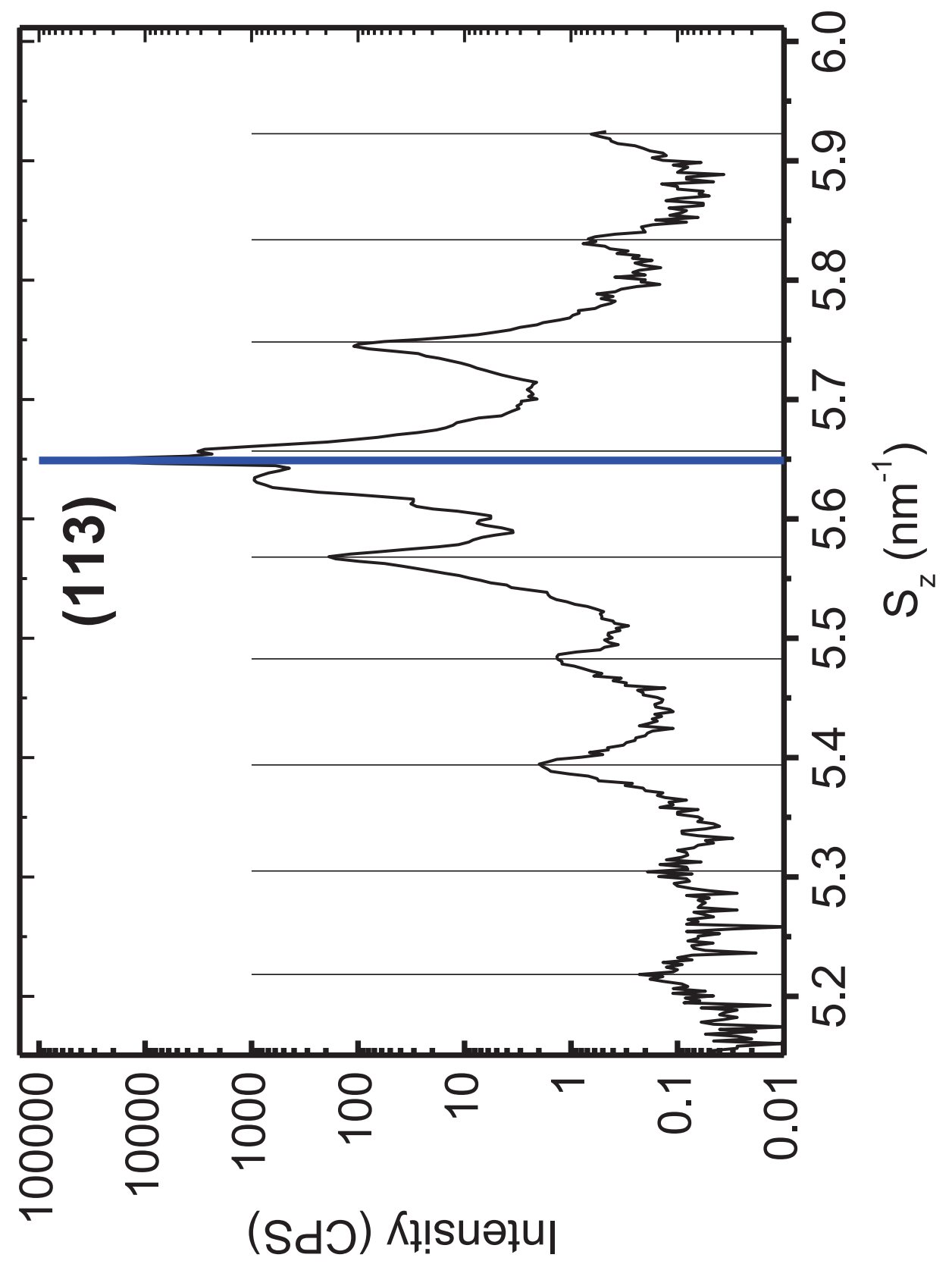




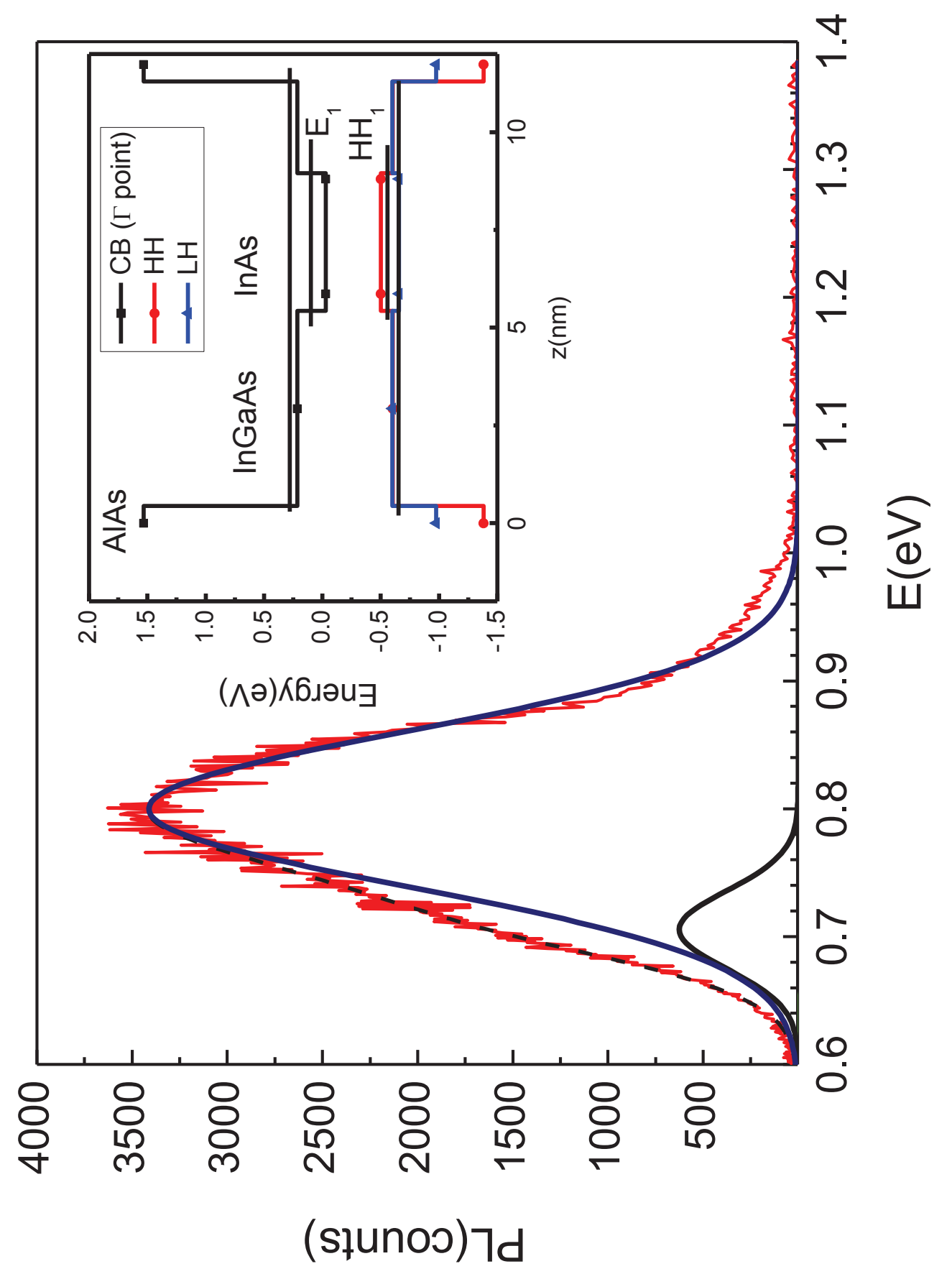




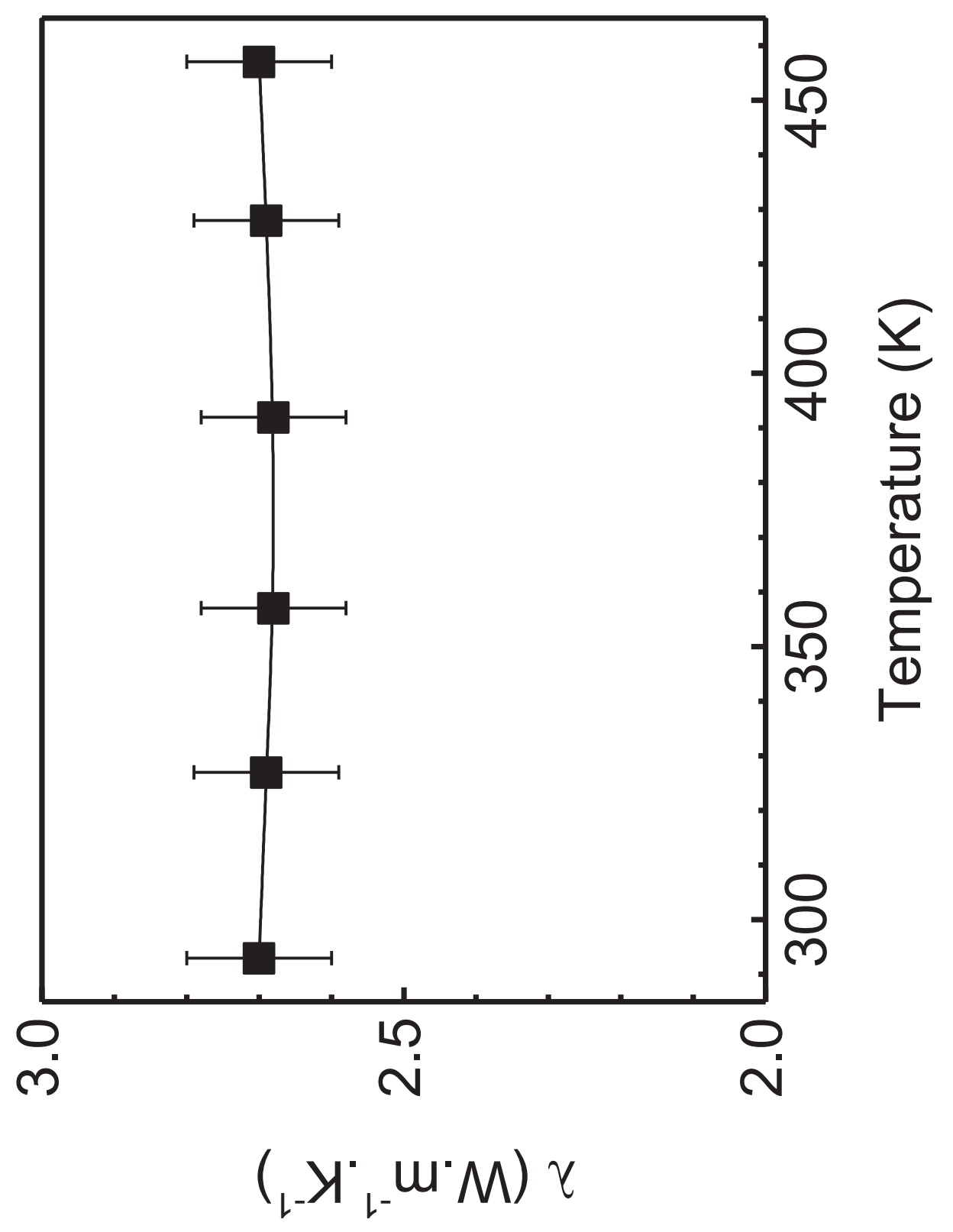

